\begin{tabular}{lll}
\multicolumn{2}{l}{ Abstract PWE-073 } & Table \\
\hline & At diagnosis & When a-TNF started \\
\hline Inflammatory & 75 & 40 \\
Fibrotic/Stricture & 25 & 51 \\
Fistula & 5 & 13 \\
Perianal only & 5 & 7 \\
Inflammatory+perianal & 10 & 11 \\
Stricture+perianal & 0 & 7 \\
Unknown/unclear & 16 & 6 \\
\hline
\end{tabular}

$58 \%$ of patients had already had surgery for CD prior to commencement of a-TNF, $13 \%$ had major abdominal surgery after a-TNF. The number of patients with stricturing disease had doubled from diagnosis to the time of a-TNF commencement. $7 / 23(30 \%)$ required surgery in less than 6 months of commencement of anti-TNF, 10/23 (43\%) in under 12 months.

Conclusion a-TNF is being commenced late in the disease process, with significant accumulated damage and $73 \%$ of ongoing surgical requirements occurring in less than 1 year after commencing a-TNF. This may be a reflection of current prescription prohibition. What remains to be demonstrated is whether earlier intervention could have prevented the large amount of intraabdominal surgery in these high risk cohorts.

Disclosure of Interest None Declared.

\section{PWE-074 TISSUE INHIBITOR OF METALLOPROTEINASE (TIMP)-3 REDUCES PRO-INFLAMMATORY CYTOKINE PRODUCTION BY ULCERATIVE COLITIS MUCOSA CULTURED EX VIVO}

IM Bell ${ }^{*}$, F Ammoscato, P Biancheri, R Curciarello, T Macdonald. CIID, Blizard Institute, Barts and The London School of Medicine and Dentistry, London, UK

\subsection{6/gutjnl-2014-307263.334}

Introduction Interleukin (IL)-1b, IL-6, IL-8 and tumour necrosis factor (TNF)-a, are elevated in the inflamed mucosa of patients with ulcerative colitis and play a central role in driving the proinflammatory immune response in this condition. TNF-a is cleaved from the cell surface by TNF-a converting enzyme (TACE), which is inhibited by tissue inhibitor of Metalloproteinase (TIMP)-3. We studied whether the addition of TIMP-3 to inflamed colonic biopsies from ulcerative colitis patients reduced the release of the pro-inflammatory cytokines TNF-a, IL-1b, IL6 and IL-8.

Methods Colonic biopsies were obtained from macroscopically inflamed areas of 4 patients with ulcerative colitis undergoing colonoscopy for a disease flare. Biopsies were then cultured ex vivo for $24 \mathrm{~h}$ in $300 \mathrm{ul}$ of serum free HL-1 medium in the absence or presence of recombinant human TIMP-3 $(100 \mathrm{ng} / \mathrm{ml})$. The concentration of TNF-a, IL-1 $\beta$, IL- 6 and IL- 8 were measured in culture supernatants by ELISA.

Results Culture with TIMP-3 significantly reduced TNF-a production by inflamed ulcerative colitis biopsies cultured ex vivo (from $1365 \mathrm{ug} / \mathrm{ml}$ in the absence of TIMP-3 to $45 \mathrm{ug} / \mathrm{ml}$ after TIMP-3 addition). Furthermore, the addition of TIMP-3 significantly reduced IL-1b production by inflamed ulcerative colitis biopsies (from 776 to $261 \mathrm{ug} / \mathrm{ml}$ ). There was a trend in the reduction of IL-6 (from 3018 to $2702 \mathrm{ug} / \mathrm{ml}$ ), which did not reach statistic significance, and no significant change in IL-8 production (from 30812 to $29114.5 \mathrm{ug} / \mathrm{ml}$ ).
Conclusion TIMP-3 administration not only causes a reduction in TNF-a via TACE inhibition but also IL-1 $\beta$. This raises the possibility of its use therapeutically in the treatment of ulcerative colitis.

Disclosure of Interest None Declared.

\section{PWE-075 ANTI-TNF THERAPY REDUCES IONISING RADIATION EXPOSURE IN PATIENTS WITH ULCERATIVE COLITIS}

D Aggarwal, JK Limdi*. Gastroenterology, Pennine Acute Hospitals NHS Trust, Manchester, UK

\subsection{6/gutjnl-2014-307263.335}

Introduction Patients with Ulcerative Colitis [UC] may be exposed to ionising radiation for evaluation of disease with inherent risks from protracted exposure. Meanwhile, bolder definitions of disease control with evolving treatment paradigms have led to earlier introduction of biological therapy. Our aim was to compare the effective radiation dose prior to and a year and 3 years after initiating anti-TNF therapy or corticosteroid in patients with UC.

Methods We performed a retrospective review of UC patients treated with anti-TNF therapy (infliximab or adalimumab) or corticosteroids at our institution from 2005 to 2013. Clinical data (demographics, disease characteristics, treatment) were obtained from case notes and electronic patient records. All instances of imaging in the previous year, 1 and 3 years after initiation of anti-TNF therapy were recorded. The effective and cumulative radiation doses were calculated from published tables [Royal College of Radiologists, UK].

Results We analysed 102 patients with ulcerative colitis (66 antiTNF and 36 corticosteroid treated). In the anti-TNF group, 68\% were males (median age $47 \mathrm{yrs}$; range 25-76; mean disease duration 9.5 yrs). Forty seven per cent had left sided disease [Montreal E2] and 55\% had pancolitis [Montreal E3]. In the corticosteroid treated patients, 55\% were males (median age 51 yrs; range 17-90; mean disease duration 7.7 yrs). Montreal classification of disease was E1 in 11\%, E2 in 46\% and E3 in 43\% respectively.

The anti-TNF cohort had a significant reduction in the number of imaging studies $(4.0$ vs. $1.5, \mathrm{p}<0.0001)$ and cumulative radiation dose $(4.1$ vs. $1.1 \mathrm{mSv}, \mathrm{p}<0.0001)$ a year after treatment. The corticosteroid group had no significant change in the number of imaging studies $(1.9$ vs. $1.3, \mathrm{p}=0.1$ or cumulative radiation dose (3.2 vs. $2.0 \mathrm{mSv}, \mathrm{p}=0.5)$.

After 3 years of anti-TNF $(n=22)$, there was a reduction in the cumulative radiation dose $(1.6$ vs. $1.0 \mathrm{mSv}, \mathrm{p}=0.3)$ and number of imaging studies $(2.7 v s .1 .9, \mathrm{p}=0.3)$. In the corticosteroid group, there was a reduction noted in the cumulative radiation dose $(2.5$ vs. $1.1 \mathrm{mSv}, \mathrm{p}<0.3)$ and no change in the number of imaging studies (1.6 vs. $1.7, \mathrm{p}=0.5$ ).

Linear regression analysis showed a decrease in cumulative radiation dose by $3.1 \mathrm{mSv}(\mathrm{CI}=-4.9$ to $+0.2, \mathrm{p}=0.07)$ and number of $\mathrm{CT}$ imaging scans by $1(\mathrm{CI}=+1.5$ to $-1.2, \mathrm{p}<0.08)$ in the anti-TNF group compared to the corticosteroid group within a year of therapy after adjusting for age, gender, disease duration, disease location and disease behaviour.

Conclusion Anti-TNF therapy is associated with a significant reduction in cumulative radiation dose and diagnostic imaging studies a year after anti-TNF therapy but not with corticosteroids. The decrease in radiation dose exposure in both groups was similar three years after treatment.

Disclosure of Interest None Declared. 